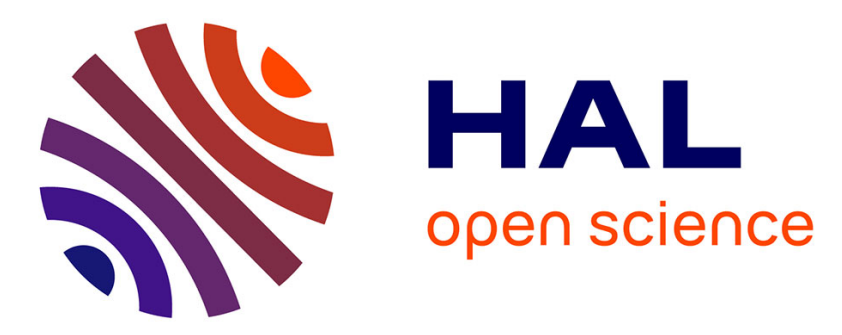

\title{
Coincident ion acceleration and electron extraction for space propulsion using the self-bias formed on a set of RF biased grids bounding a plasma source
}

D.V. Rafalskyi, Ane Aanesland

\section{- To cite this version:}

D.V. Rafalskyi, Ane Aanesland. Coincident ion acceleration and electron extraction for space propulsion using the self-bias formed on a set of RF biased grids bounding a plasma source. Journal of Physics D: Applied Physics, 2014, 47 (49), pp.495-203. 10.1088/0022-3727/47/49/495203 . hal-01102846

\section{HAL Id: hal-01102846 \\ https: / hal.sorbonne-universite.fr/hal-01102846}

Submitted on 13 Jan 2015

HAL is a multi-disciplinary open access archive for the deposit and dissemination of scientific research documents, whether they are published or not. The documents may come from teaching and research institutions in France or abroad, or from public or private research centers.
L'archive ouverte pluridisciplinaire HAL, est destinée au dépôt et à la diffusion de documents scientifiques de niveau recherche, publiés ou non, émanant des établissements d'enseignement et de recherche français ou étrangers, des laboratoires publics ou privés. 


\title{
Coincident ion acceleration and electron extraction for space propulsion using the self-bias formed on a set of RF biased grids bounding a plasma source
}

\author{
D. Rafalskyi and A. Aanesland
Laboratoire de Physique des Plasmas (CNRS, Ecole Polytechnique, Sorbonne Universités, UPMC Univ Paris 06, Univ Paris-Sud), \\ Ecole Polytechnique, 91128 Palaiseau, France
}

\begin{abstract}
We propose an alternative method to accelerate ions in classical gridded ion thrusters and ion sources such that co-extracted electrons from the source may provide beam space charge neutralization. In this way there is no need for an additional electron neutralizer. The method consists of applying RF voltage to a two-grid acceleration system via a blocking capacitor. Due to the unequal effective area of the two grids in contact with the plasma, a DC self-bias is formed, rectifying the applied RF voltage. As a result, ions are continuously accelerated within the grid system while electrons are emitted in brief instants within the RF period when the RF space charge sheath collapses. This paper presents the first experimental results and a proof-ofprinciple. Experiments are carried out using the Neptune thruster prototype which is a gridded Inductively Coupled Plasma (ICP) source operated at $4 \mathrm{MHz}$, attached to a larger beam propagation chamber. The RF power supply is used both for the ICP discharge (plasma generation) and powering the acceleration grids via a capacitor for ion acceleration and electron extraction without any DC power supplies. The ion and electron energies, particle flux and densities are measured using retarding field energy analyzers (RFEA), Langmuir probes and a large beam target. The system is operating in Argon and $\mathrm{N}_{2}$. The DC self-bias is found to be generated within the gridded extraction system in all the range of operating conditions. Broad quasi-neutral ion-electron beams are measured in the downstream chamber with energies up to $400 \mathrm{eV}$. The beams from the RF acceleration method are compared with classical DC acceleration with an additional external electron neutralizer. It is found that the two acceleration techniques provide similar performance, but the ion energy distribution function from RF acceleration is broader while the floating potential of the beam is lower than for the DC accelerated beam.
\end{abstract}

\section{Introduction}

Since the early 1990s, broad-beam ion sources (IS) have been used as thrusters for commercial satellites and engines for scientific space missions. Using IS in space thruster applications require neutralization or compensation of both the ion positive space charge and the ion current [1]. Commonly, electrons are injected from an external neutralizer into the downstream beam. The neutralizer is a dedicated device based on thermo-electron emission or different kinds of gas discharges that usually needs additional power supplies and gas injection systems. Although much progress on the neutralizer technology has been achieved over the last few years, they are still considered as the part of the thruster with shortest lifetime and they are fragile during launch [1-3].

Simultaneous emission of oppositely charged particles from a single source [4-11] may provide several advantages in space. The additional neutralizer is then redundant, reducing the corresponding power and efficiency losses in the thruster and subsystems etc. Concepts using magnetic nozzles [8] or double layer structures formed at the plasma source output allow the acceleration of a quasi-neutral plasma [7,9]. Though these concepts provide in principle long-life neutralizer-free operation, the achievable performance such as specific impulse and thrust efficiency are generally lower than the performances provided by the traditional electric propulsion concepts (ion- and Hall-thrusters) [7,9,12,13]. Concepts based on simultaneous extraction of positive ions and electrons [4-9] or positive and negative ions [10,11] accelerated via biased grids may also provide a quasi-neutral beam. One advantage of these systems is the 
similarity with space proven gridded thrusters allowing technology heritage. The PEGASES thruster is a gridded ion-ion thruster that accelerated alternately positive and negative ions for thrust [10]. Operation of the PEGASES source is possible only with electronegative gases, i.e. halogens, and the best propellant for this thruster is Iodine [11]. Therefore, for operation with Xe or other inert gases is not possible with this concept. Ions and electrons have been co-extracted from a single-grid source where the grid is biased with RF frequency, and this concept was also proposed for electric propulsion $[4,14]$. In the latter case, a so-called RF plasma self-bias effect is used for acceleration and co-extraction of electrons [15]. This source provides a separate control of the ion energy and flux of the broad quasi-neutral beam. However, it was shown that the source could not operate with ion energies of more than 200-300 eV and the ion extraction efficiency remained low [16]. As a result, this concept was not developed further for space applications.

We propose here an alternative method to accelerate ions using the "RF self-bias" effect. Briefly, the idea is to apply an RF bias voltage to a two-grid acceleration system via a blocking capacitor. In this case the space charge sheath in front of the grids oscillates with the RF frequency. The blocking capacitor charges up as a result of the effective area ratio between the first and second grid being larger than one. This allows rectification of the applied RF voltage by plasma and formation of a DC self-bias. In this system ions are continuously accelerated within the grids while electrons are emitted in brief instants within the RF period when the RF space charge sheath collapses.

This paper presents the first experimental results and proof-of-principle. The paper is organized as follows. In section II, the RF acceleration concept is presented with a detailed discussion on the RF self bias in a gridded system. The experimental setup is presented in section III, results are discussed in section IV and a conclusion is drawn in section V.

\section{The Neptune RF acceleration concept}

RF powered electrodes in asymmetric capacitive coupled plasma sources are widely used in the semiconductor industry to control independently the ion flux and ion energy impinging on the biased surface $[15,17,18]$. The RF voltage drop across the sheath in front of the driven and grounded surfaces is inversely proportional to the sheath capacitance, $C=\varepsilon_{0} A / d$, where $A$ and $d$ are the sheath surface and thickness, respectively, and $\varepsilon_{0}$ is the vacuum permittivity. Hence, the largest voltage drop is across the smaller electrode. When this driven electrode is connected to ground via a blocking capacitor (avoiding DC currents to ground [15]), a DC bias is formed that rectify the RF voltage drop across the sheath. The self-bias formed across the low pressure sheath is typically $V_{d c} \sim 0.78 V_{r f}$, where $V_{r f}$ is the amplitude of the RF voltage applied to the electrode [15]. As a result, the ions are accelerated continuously across the combined DC-RF sheath, while electrons escape during the short RF period where RF voltage approaches the plasma potential and the sheath collapses $[4,14,17,18]$. The average ion and electron currents to the electrode are equal due to the presence of the blocking capacitor in the circuit. Due to the RF oscillating sheath, the ion energy distribution impinging on the electrode surface tends to be bimodal with an energy spread decreasing with increasing RF frequency [15]. Replacing the smaller driven electrode by a grid allows the generation of coincident ion-electron flow, as shown in a single-grid system [4]. As mentioned in the introduction, single-grid systems are strongly limited by grid sputtering and low source efficiency [16].

In this paper we propose to apply only the RF voltage to a set of grid in a "classical" gridded ion thruster or ion source. In the following we will elaborate how this may accelerate positive ions creating thrust, with co-extracted electrons provide space charge neutrality. Note, that we call this new concept "Neptune" after a dedicated research project acronym [19].

Figure 1 shows a simplified drawing of the grid system and an equivalent RF electric circuit in line with the one of Coburn and Kay [17]. The RF voltage is applied between the first and second grid. The first grid is directly in contact with the plasma while the second grid is placed downstream and seen by the plasma only through the holes in the first grid. Oscillating 
space charge sheaths, represented by a capacitor and diode in parallel, are formed between the plasma and first grid, and between the plasma and second grid. The diodes in the circuit appears due to the asymmetry in the time response between the heavy ions and the much lighter electrons to the applied electric field, where the electron motion is controlled by the instantaneous electric field while the ion motion is mainly defined by the average electrical field. The sheath capacitance between the plasma and the first grid $C_{1}$ is higher than the sheath capacitance between the plasma and the second gird $C_{2}$ since the second grid is partially screened by the first one, i.e. the effective area ratio between the first and second grid is larger than one. As a result, the applied RF voltage $V_{R F}$ mainly drops across the sheath formed in front of each aperture of the second grid. The RF voltage is rectified to a DC self-bias voltage $V_{D C}$ due to the mentioned asymmetry in time response between ions and electrons and due to blocked DC current by blocking capacitor. Thus, in this case the asymmetry or difference in the DC self-bias voltage is formed between the extraction grids (external to the main plasma source).. The DC component then accelerates ions continuously while electrons are expelled in moments when the oscillating plasma potential approaches zero and the inter-grid sheath collapses. Figure 2 shows the expected RF plasma potential waveform with respect to the grounded second grid. A $\varphi_{c r}$ is the plasma potential corresponding to the sheath collapse, and can be estimated using the Child's law for the given aperture dimensions and ion flux from plasma $[15,16]$.

As for RF biased electrodes, the ion energy distribution depends strongly on the applied RF frequency [20]. For the self-bias to form, the applied RF frequency $\omega$ must be situated between the ion $\omega_{\mathrm{pi}}$ and electron plasma frequency $\omega_{\mathrm{pe}}$ defined as:

$$
\omega_{p e}=\sqrt{\frac{e_{0}^{2} n_{e}}{\varepsilon_{0} m_{e}}}, \quad \omega_{p i}=\sqrt{\frac{e_{0}^{2} n_{e}}{\varepsilon_{0} M_{i}}},
$$

here $e_{0}$ is the electron charge, $n_{e}$ is the plasma density, $\varepsilon_{0}$ is the vacuum permittivity and $M_{i}$ is the ion mass. Usually, this range includes RF signals from a few to hundreds of $\mathrm{MHz}$, where the ion energy spread is inversely proportional to the applied frequency [15].

(a)

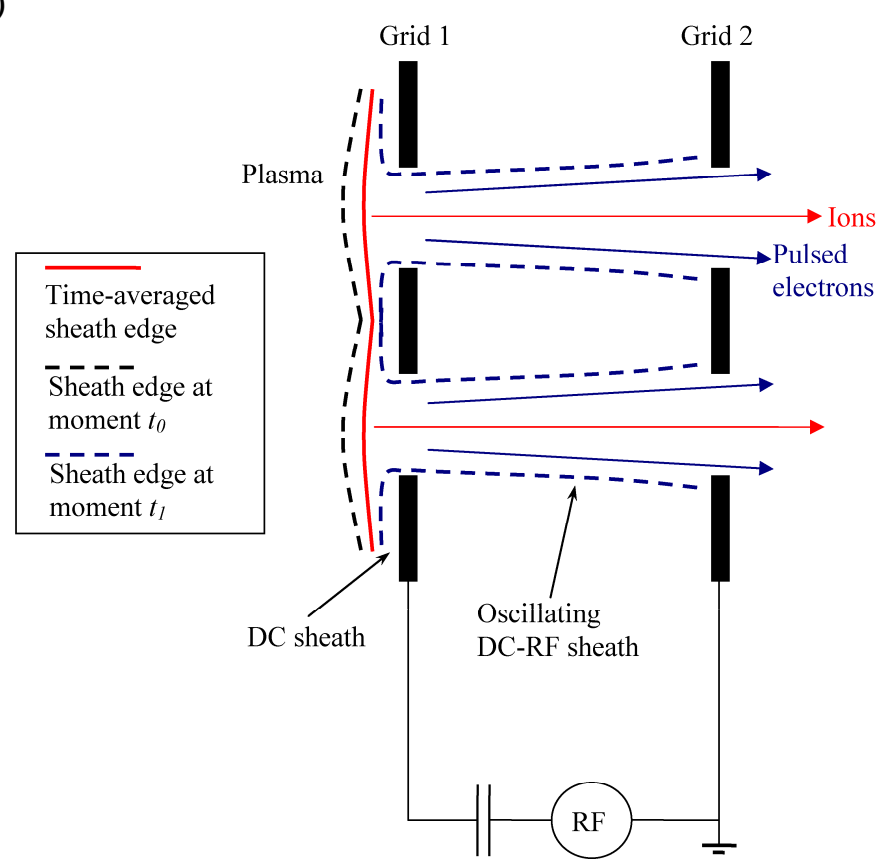


(b)

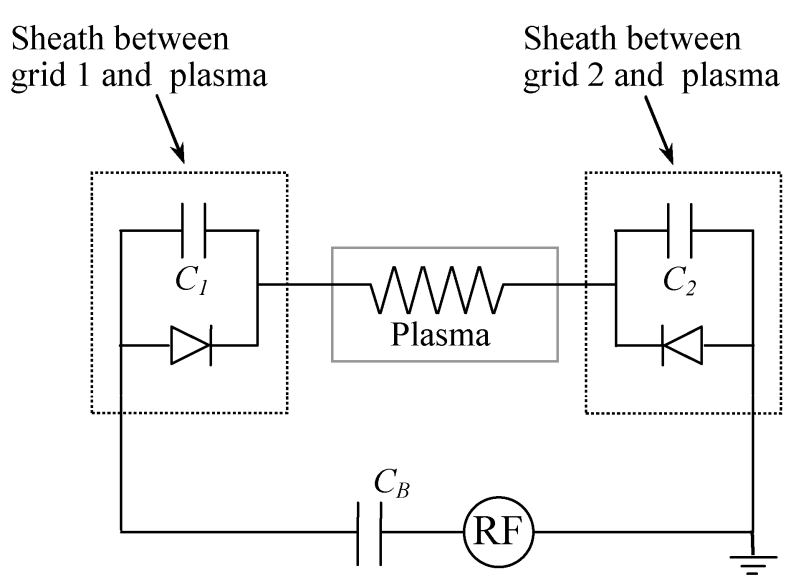

Fig. 1. (a) Schematic view of a two beamlets in the two-grid extraction system when an RF voltage is applied between the grids, $t_{1}$ and $t_{2}$ corresponds to those on the Fig. 2 below; (b) equivalent circuit of the two extraction grids powered by an RF voltage.

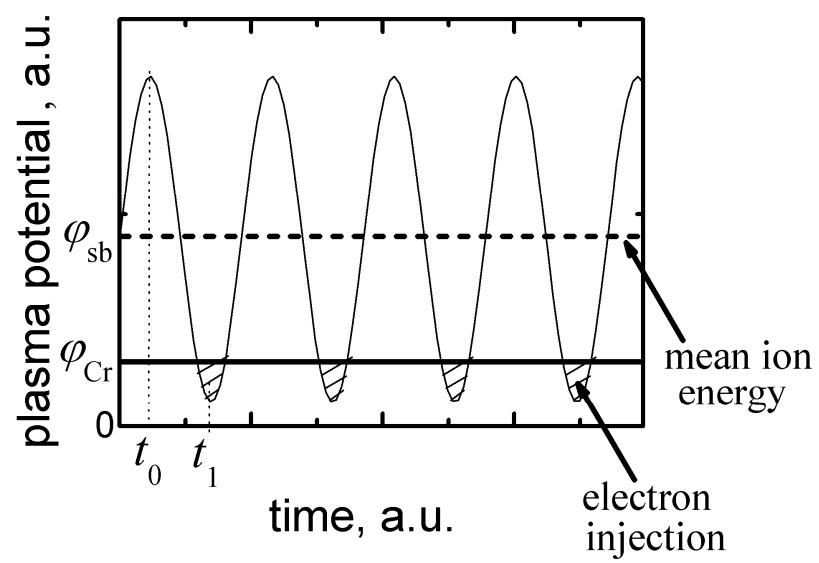

Fig. 2. The plasma potential waveform during the simultaneous extraction of ions and electrons from the two-grid RF-biased source. The plasma potential is shown with respect to the grounded second grid. $\varphi_{c r}$ is the plasma potential corresponding to the sheath collapse, and can be estimated using the Child's law for the given aperture dimensions and ion flux from plasma $[15,16]$.

\section{Experimental setup}

The experiments are carried out using an inductively coupled plasma source attached to a larger beam propagation chamber illustrated in figure 3. This system has been detailed previously $[21,22]$, however the gridded thruster used here is a modification of the PEGASES thruster prototype II [16]. We call the modified RF thruster "Neptune". Due to the partly thruster heritage, the first prototype has a rather unconventional geometry compared to classical gridded thrusters. The source is a rectangular parallelepiped with size of $8 \times 12 \times 12 \mathrm{~cm}$. The plasma is generated using an inductively coupled plasma (ICP) discharge (see ref [23] for details) powered by a 4 $\mathrm{MHz} \mathrm{RF}$ generator. A set of two grids is placed at the source exit. In this particular Neptune source, an RF distribution and matching system (see Fig.3) is introduced to allow matching of the RF generator with the plasma load and to distribute the RF power between the ICP inductor 
and extraction grid system. The first grid in contact with the plasma is biased with RF voltages via a blocking capacitor and the applied RF voltage amplitude is controlled using the RF power distribution system in the range 0-600 Vp-p. The second grid is grounded. The DC component of the first grid voltage is measured using both an oscilloscope and a 24 bit ADC board equipped with high RF impedance filters and resistive dividers. Note here, that the grids are called the "first" and "second" grid referring to the position from the plasma source; in the literature they may also be referred to as the "screen" and "acceleration" grids, respectively. Both grids are made of stainless steel and have an optical transparency of 0.6 and an aperture diameter of 2.5 $\mathrm{mm}$; the intergrid distance is $2 \mathrm{~mm}$. The extraction system is a rectangular with dimensions $65 \times 105 \mathrm{~mm}$. The aperture size in the extraction system ensures periodical electron sheath collapse when time-oscillating plasma potential is below approximately $95 \mathrm{~V}$ (relative to the grounded second grid), according to estimation using Child's law [15,16]. At this potential space charge sheath thickness becomes equal to the radius of the apertures in the extraction grid allowing plasma expansion through the extraction system. The RF power is continuously measured for both the ICP and extraction channels separately, so it is possible to operate the source with constant ICP power while changing the RF voltage applied to the extraction system. Due to the experimental heritage the design of this prototype has not been optimized in the view of gas utilization and efficiency requirements, and is used in this work only to demonstrate a proof-of-principle.

A Langmuir probe and a planar probe are introduced into the prototype through the grids in order to measure the plasma density and the ion flux to the extraction system. The experiments presented here were performed at a constant ion current density to the extraction system of about $1 \mathrm{~mA} / \mathrm{cm}^{2}$, which corresponds to approximately $150 \mathrm{~W}$ of RF power absorbed by the ICP channel with Ar gas at standard working pressure (see below). At this set of parameters, the ion plasma frequency $\omega_{\mathrm{pi}} / 2 \pi$ is approximately $4 \mathrm{MHz}$, which is equal to the RF generator frequency and therefore corresponds to the lower frequency limit allowing the DC self-bias to form according to eq. (1). The Neptune thruster prototype is connected to a $1 \mathrm{~m}$ long, $70 \mathrm{~cm}$ diameter beam propagation chamber. The chamber is equipped with a $15 \mathrm{~cm}$ diameter beam target, a retarding field energy analyzer (RFEA) and an RF-compensated Langmuir probe. The Langmuir probe and the RFEA can be moved perpendicular and parallel to the beam propagation, while the beam target can only be moved in the axial (parallel) direction. The RF compensation of the Langmuir probe is made using a reference electrode connected to the probe tip via a capacitor and inductor chokes connected in series with the probe acquisition system, allowing the probe potential to follow RF fluctuations in the beam-plasma. The reference electrode serves also as a probe tube holder and has a total area of about $5 \mathrm{~cm}^{2}$. RF oscillations in the beam are measured using the reference electrode connected to an oscilloscope with a low-impedance line. In order to compare the Neptune beam parameters with parameters of an ion beam generated in a classical way (using DC ion acceleration with an external neutralizer) a hot cathode neutralizer can be introduced into the beam chamber. In this case, the first grid is disconnected from the RF system and connected to a DC voltage source.

The gas is fed into the Neptune prototype via a shower-like injection system. Ar is used as a working gas, unless stated otherwise. The Ar flow rate is $25 \mathrm{sccm}$. The pressures in the discharge chamber and beam chamber are about 1.5 mTorr and 0.2 mTorr, respectively. The beam chamber is pumped using a turbo pump in connection with a dry pump. In total, the various experiments using the Neptune RF extraction system were running for about 15 hours. The measured currents and fluxes are repeated with a variation less than $\pm 10 \%$, and include heating and cooling effects of the RF system, drift in the mass-flow controller, etc. 


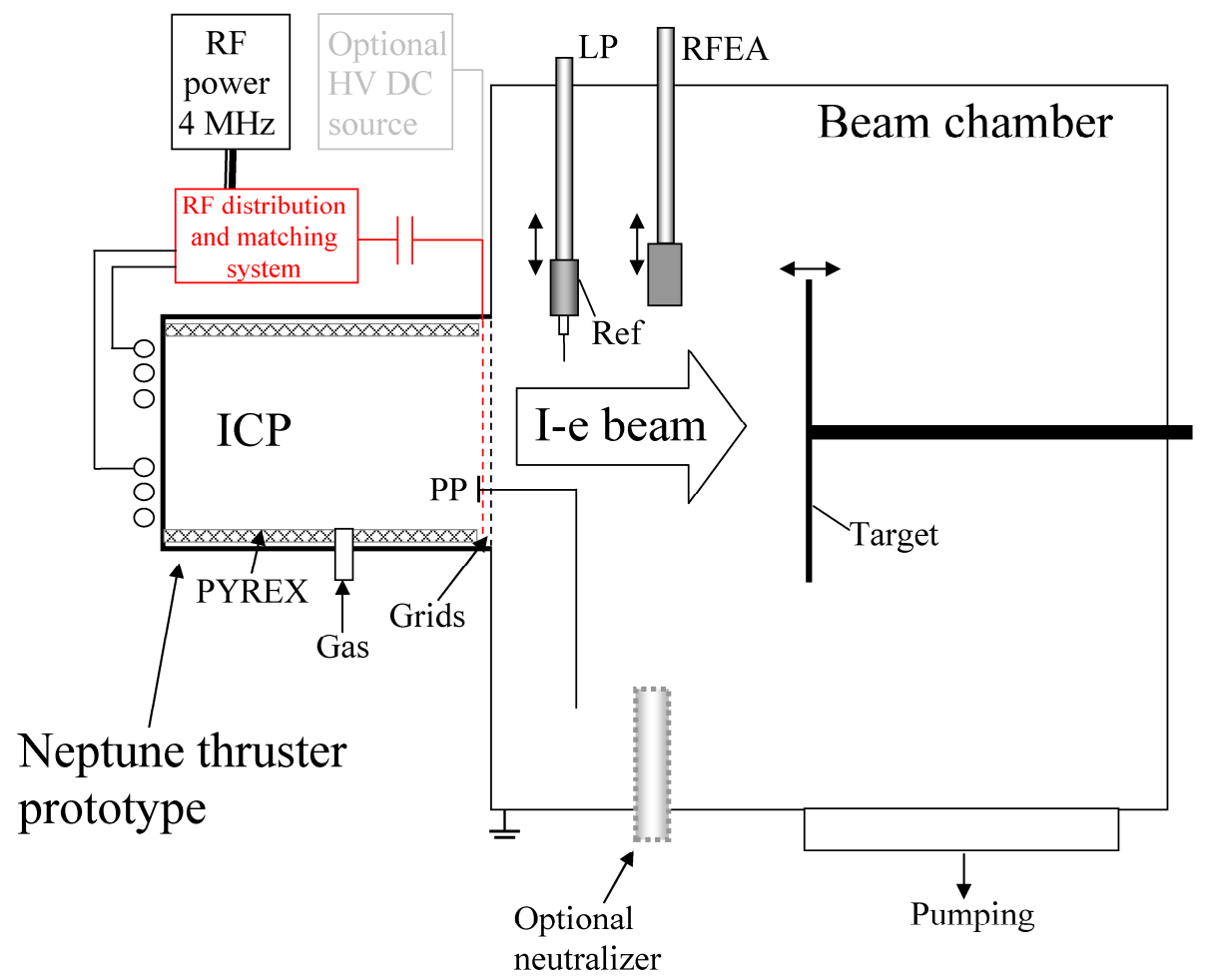

Fig. 3. Schematic of the experimental setup. The "LP" is the Langmuir probe, the "PP" is the planar probe and the "Ref" is the reference electrode, connected capacitively to the "LP" tip. In the RF mode the neutralizer and DC voltage source (shown in grey on the figure) are not included in the experimental system. The neutralizer and DC source are installed and turned on only in the experiments with DC ion extraction (DC mode).

\section{Experimental results}

The first tests of the Neptune prototype operating with Ar is carried out by measuring the RF and DC voltages formed on the first grid as a function of the applied RF voltage. Fig. 4 (a) shows a typical voltage waveform measured on the first grid. The offset of about $200 \mathrm{~V} \mathrm{DC}$ shows that the RF voltage is rectified and that a DC self-bias is formed. Fig. 4 (b) shows the measured DC component of the grid voltage $V_{D C}$ as a function of the amplitude of the applied RF voltage $V_{R F}$. It is seen that $V_{D C} \approx 0.8 V_{R F}$, which is in agreement with theoretical estimations for the self-bias effect in low-pressure plasmas with asymmetrical electrodes [15].
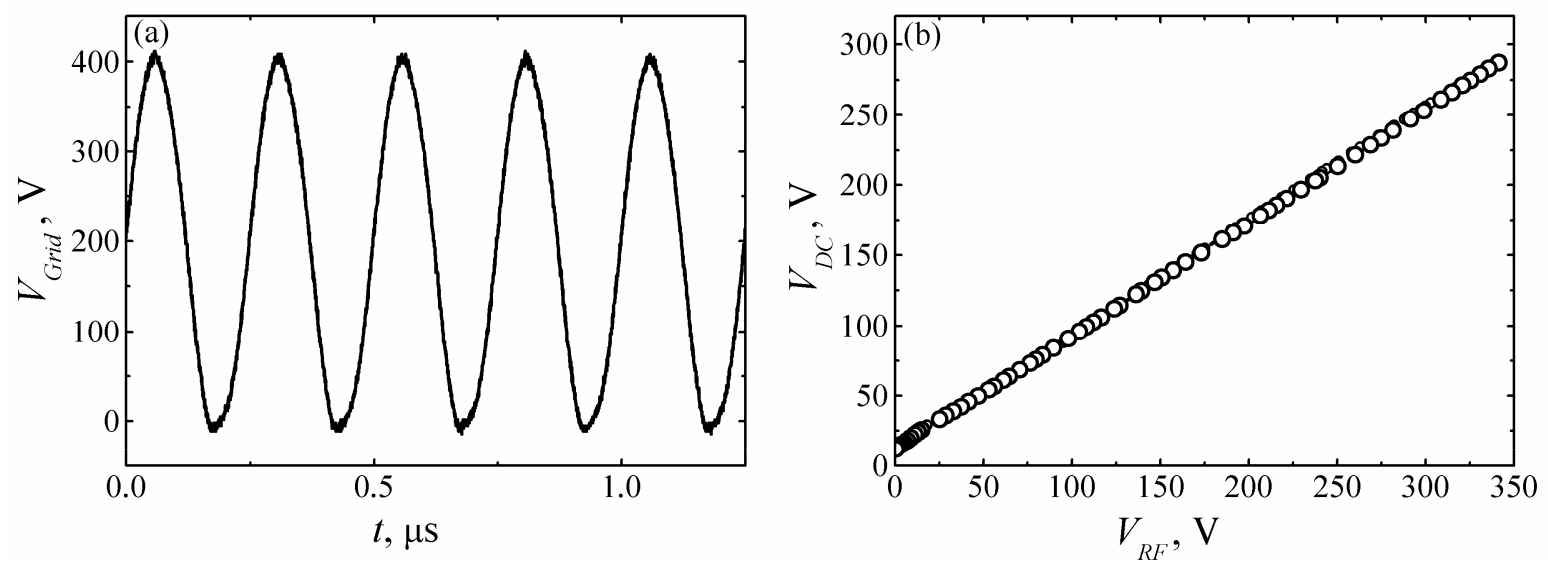

Fig.4. (a) Voltage waveform measured on the RF-powered first grid of the Neptune source; the applied RF voltage amplitude $V_{R F}$ is $200 \mathrm{~V}$. (b) DC self-bias voltage $V_{D C}$ in the extraction system measured as a function of the amplitude of the applied RF voltage $V_{R F}$. 
The formation of a DC self-bias allows a continuous ion extraction/acceleration from the Neptune prototype. Fig. 5 shows the measured ion beam current as a function of the DC self-bias voltage. It is seen here that the extracted current continuously increases with increasing DC selfbias voltage reaching $16 \mathrm{~mA}$ at about $300 \mathrm{~V}$ of $V_{D C}$. To evaluate this result, the RF acceleration technique is compares with the extracted ion current obtained using a traditional gridded thruster configuration, i.e. in this case the neutralizer is switched "on" and only a DC voltage is applied between the extraction grids. Below we will call this the "DC-mode" of operation and the RF extraction without neutralizer the "RF-mode".

The ion beam current measured as a function of the applied DC voltage (from the DCmode) is also plotted on figure 5. Comparing the two results shows that the ion extraction in the RF-mode is about 20\%-30\% more efficient than in the DC-mode. This is in agreement with previous observations in the single-grid RF sources $[4,16]$. The reason for this effect may be due to the difference in the effective space charge sheath thickness between a pure DC sheath and a $\mathrm{DC}+\mathrm{RF}$ sheath due to the oscillating nature of the RF sheath [15], hence a change in the ion optics. Note however that the extraction system has not yet been optimized for the given acceleration voltage $(0-400 \mathrm{~V})$ and ion current density from the plasma $\left(1-1.5 \mathrm{~mA} / \mathrm{cm}^{2}\right)$. Therefore, the ion beam currents in both the DC and RF modes do not reach the saturation limit given by the optical grid transparency and the space charge limit. We expect that the maximum value of the extracted ion current will be similar for DC and RF ion extraction since this is only a question of optimizing the grids for the given ion flux and acceleration voltage [1].

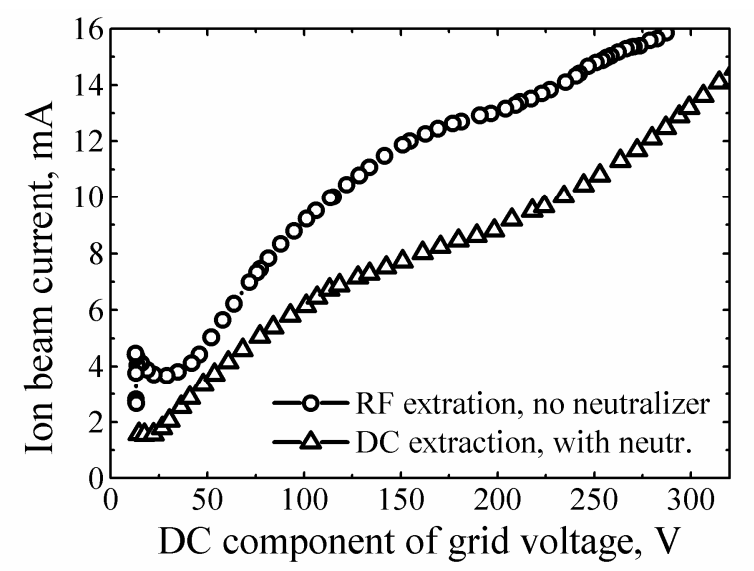

Fig.5. The ion beam current as a function of the generated DC self-bias (circles) or applied DC voltage (squares) on the extraction system. A downstream electron neutralizer is used in the DC mode.

Fig. 6 shows simultaneously measured ion and electron energy distribution functions (IEDF and EEDF, respectively) emitted from the source for two different DC self-bias voltages. The neutralizer is not used here. The ion energy distribution is multi-peaked while electrons have a single peak extracted with low energy of about $10 \mathrm{eV}$. At RF voltages above about $100 \mathrm{~V}$, the accelerated beam becomes visible (see photo on fig. 7) which may indicate excitation of neutrals and ions in the downstream region by co-extracted electrons emitted from the source. The RFEA measurements show that the total amount of ions and electrons are similar and indicates that the space charge is compensated. This will also be demonstrated from target measurements below.

The IEDF measured in the RF mode is not similar to the case of DC ion acceleration shown in fig. 8. This can be explained as follows. In theory, the IEDF of single mass ions accelerated in an RF+DC electrical field is double-peaked, where the peak separation width is a function of the applied frequency [24]. This happens when the ion transit time across the sheath is comparable to the RF period. Figure 6 show that in our case the IEDFs are multi-peaked, where the main peak roughly corresponds to the self-bias potential. Similar behavior of IEDFs has been observed in relatively high-pressure RF discharges where the space charge sheath 
becomes collisional with dominating charge-exchange collisions [25]. Our estimations using cross-sections for both ion-neutral collisions and charge-exchange processes $\mathrm{Ar}^{+}+\mathrm{Ar}=\mathrm{Ar}+\mathrm{Ar}^{+}$ $[26,27]$ indicates however that the sheaths in the Neptune prototype is collisionless in the pressure range of a few mTorr. We therefore suppose that the multi-peak behavior of the IEDF originates from the non-flat shape of the oscillating RF sheath located between the grids (in contrast to common RF plasma systems), so the ion transit time across the sheath is a function of the initial radial ion position in front of each beamlet. In any case, the ion peak broadening seen on the measured IEDF is expected to be significantly lower at higher RF frequencies, when the ion transit time across the sheath is much longer than the RF period [24].
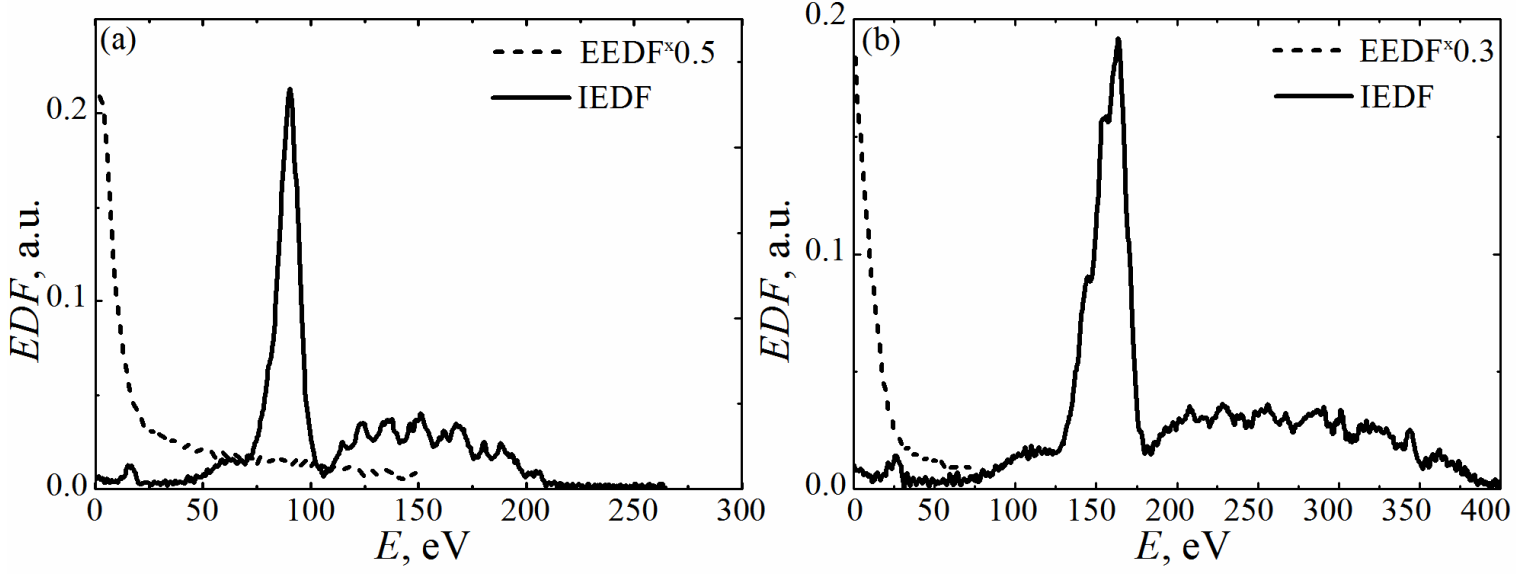

Fig.6. Energy distribution functions for the ions (IEDF, solid line) and electrons (EEDF, dashed line) measured simultaneously at the Neptune source output using $200 \mathrm{~V}$ (a) and $400 \mathrm{~V}$ (b) of peak-to-peak RF voltage applied to the extraction system. The EDFs are plotted in arbitrary units (a.u.). Fig.5 and Fig. 9 provide the corresponding fluxes.

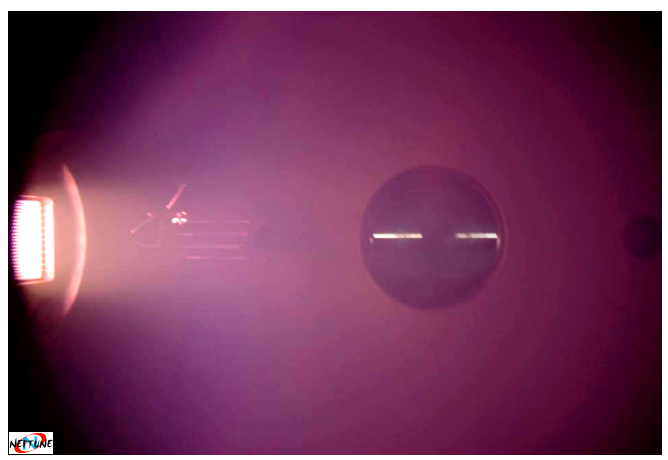

Fig. 7 A photo of the plume produced by the Neptune source operating in the RF mode with a DC self bias voltage of $200 \mathrm{~V}$.

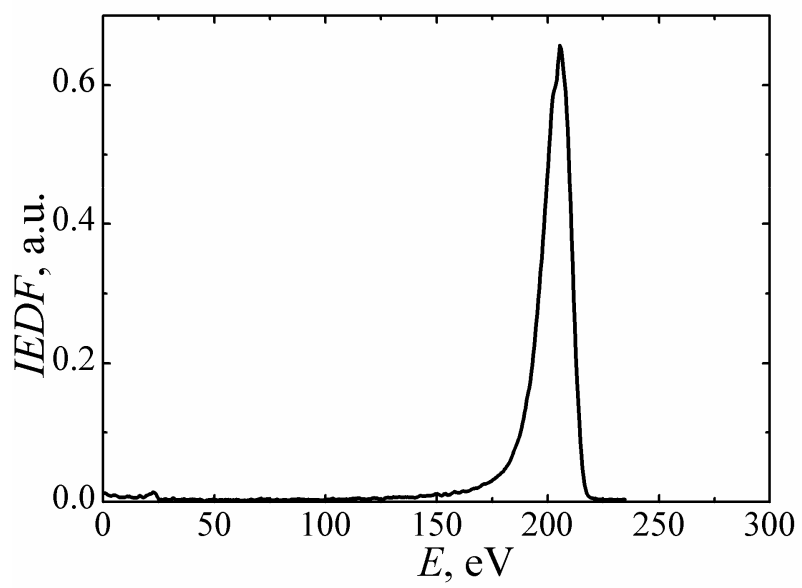


Fig. 8. Ion energy distribution function (IEDF) measured at the Neptune source output using only DC acceleration voltage $(200 \mathrm{~V})$ applied to the extraction system. The downstream neutralizer is operating in this experiment.

The spatial distribution of the ion flux from the Neptune prototype has been measured using a movable RFEA placed in the beam transport chamber $10 \mathrm{~cm}$ from the source output. Fig. 9 shows the ion flux measured in the RF and DC mode. The ion beams are well confined with quantitatively the same beam profile in both cases. Hence, in this system RF powering of the extraction system provides ion beam formation of almost the same quality as with conventional DC-bias and neutralizer. It should be mentioned here that the apparent "focusing" of the beam (i.e the beam envelope being narrower than the grid size) is most likely due to the plasma density distribution is the source rather than an "optical" beam focusing due to the grid optics.

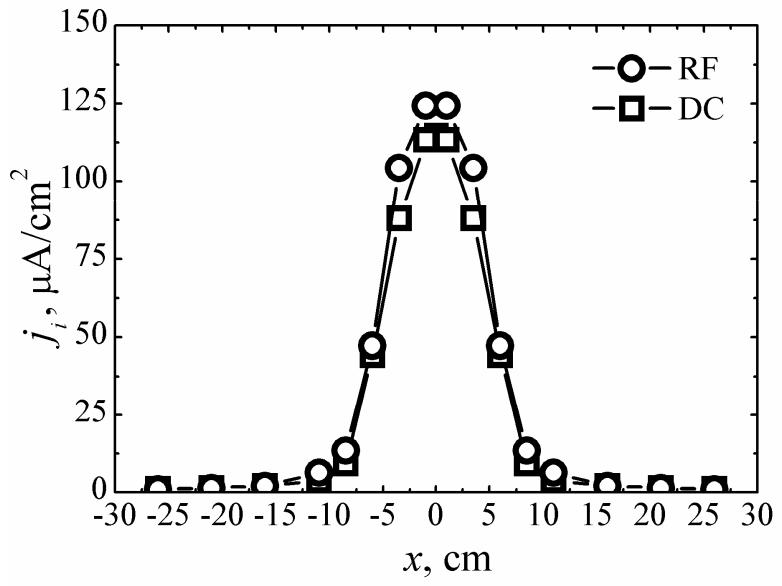

Fig. 9. The spatial distribution of the ion flux measured $10 \mathrm{~cm}$ from the Neptune output using a calibrated RFEA. Circles and squares are measured in RF and DC mode, respectively. The DC component of the acceleration voltage is $200 \mathrm{~V}$ in both cases. The center line of the extraction system is located at $x=0 \mathrm{~cm}$, the edge of the extraction system corresponds to $-5.2 \mathrm{~cm}$ $<\mathrm{x}<5.2 \mathrm{~cm}$.

While ion and electron extraction from an RF powered extraction system is demonstrated by the results shown above, the space charge compensation in the downstream beam needs to be investigated separately. This is investigated by a big planar probe (beam target) placed $10 \mathrm{~cm}$ downstream of the grids. Fig. 10 (a) shows an IV curve measured on this beam target when a 300 $\mathrm{eV}$ ion-electron beam propagates in the downstream chamber. The figure shows that both ions and electrons are extracted from the source: when the target is biased positive, with respect to the grounded beam propagation chamber, the emitted electron current is larger than the ion saturation current measured on the target. Equal ion and electron currents are achieved with a target potential of about 10 Volts, i.e. corresponding to the beam floating potential. 

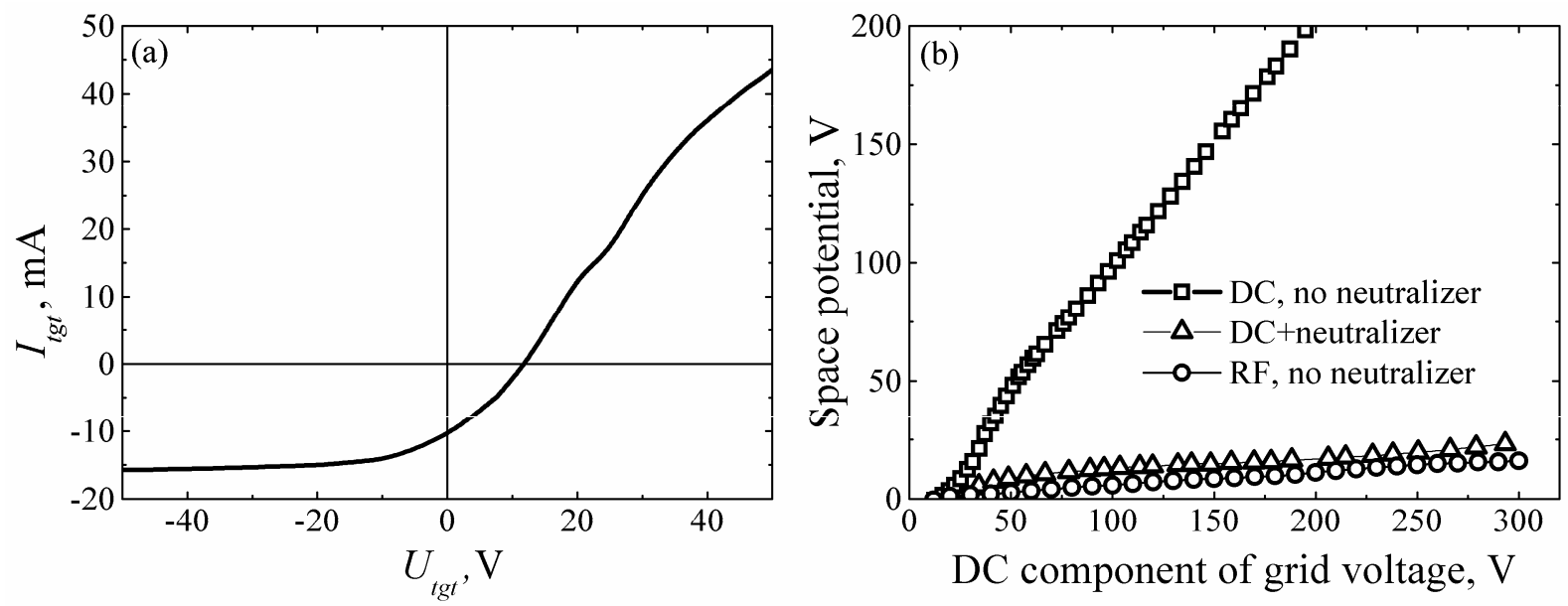

Fig. 10. (a) IV curve of the target affected by the $300 \mathrm{eV}$ ion-electron beam generated by the Neptune source. Positive and negative currents correspond to the current of negatively and positively charged particles, respectively; (b) Floating potential of the target ("Space potential") as a function of the DC component of the grid voltage, measured for DC ion extraction without (squares) and with neutralizer (triangles), and for the RF mode with i-e beam extraction without neutralizer (circles).

The floating target potential is measured as a function of the DC component of the acceleration potential and shown on Figure 10 (b) for different operation modes. The floating potential measured in the DC mode without the neutralizer follows almost the acceleration potential, showing that the ion beam space charge is not compensated by electrons and the target is charged up by the ions. The floating potential measured with the neutralizer operating at an emission current of $20 \mathrm{~mA}$ shows that the target potential in this case is almost constant with a value close to $20 \mathrm{~V}$. The floating potential increases slightly with increasing acceleration voltage. In the RF mode, the floating potential is around 10-15 V also slightly increasing with the acceleration voltage. The lower floating potential compared to the DC mode may be due to a high directionality of the emitted electrons in this case.

In the RF-mode the electrons are emitted periodically following the $4 \mathrm{MHz} \mathrm{RF}$ frequency. Therefore, the measured time-averaged properties of the beam compensation do not show the complete picture as the plume plasma potential may oscillate with a certain amplitude. In order to measure the amplitude of these oscillations, a Langmuir probe is placed on the axis of the i-e beam $10 \mathrm{~cm}$ from the source output. Fig. 11 shows the voltage waveform measured on the LP reference electrode ("Ref" at Fig. 3) together with the voltage waveform applied to the extraction system. The floating potential in the beam is non-sinusoidal with sharp local minimums corresponding to the electron injection periods. The peak-to-peak amplitude of the oscillation is however only about 5 Volts, corresponding to less than $1 \%$ of the applied RF voltage. The plume potential oscillations are therefore almost negligible. Investigation of thruster plume properties is a difficult task as vacuum facility effects plays an important role [1,28]. Also here, the plume potential oscillations are expected to be dependent on the external boundary conditions (in space this is influenced on the various reference potentials between the thruster, spacecraft body, solar panels etc. ) Thus, it is evident that the Neptune thruster should also be tested in larger vacuum facilities. 


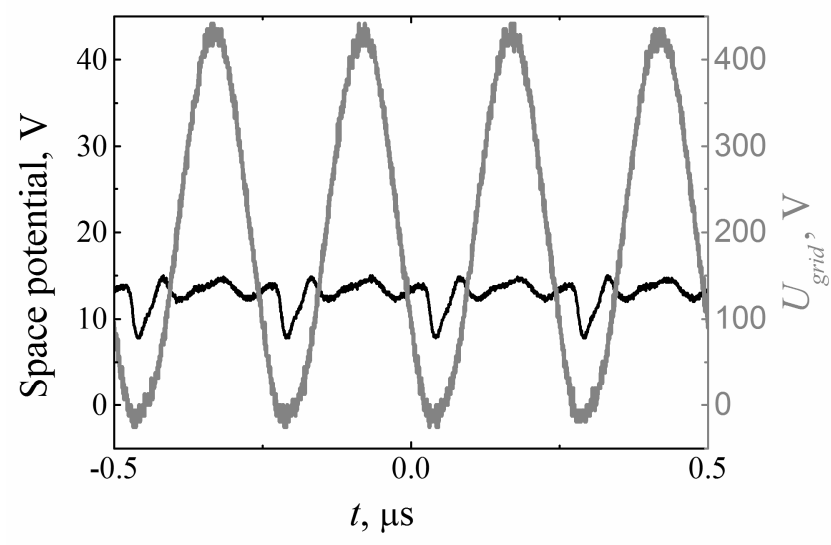

Fig. 11 Waveforms of the floating probe potential (black) and first grid potential (grey) measured simultaneously for a DC self-bias of $200 \mathrm{~V}$ formed on the extraction system, which corresponds to about $220 \mathrm{~V}$ of applied RF voltage amplitude (440 V peak-to-peak).

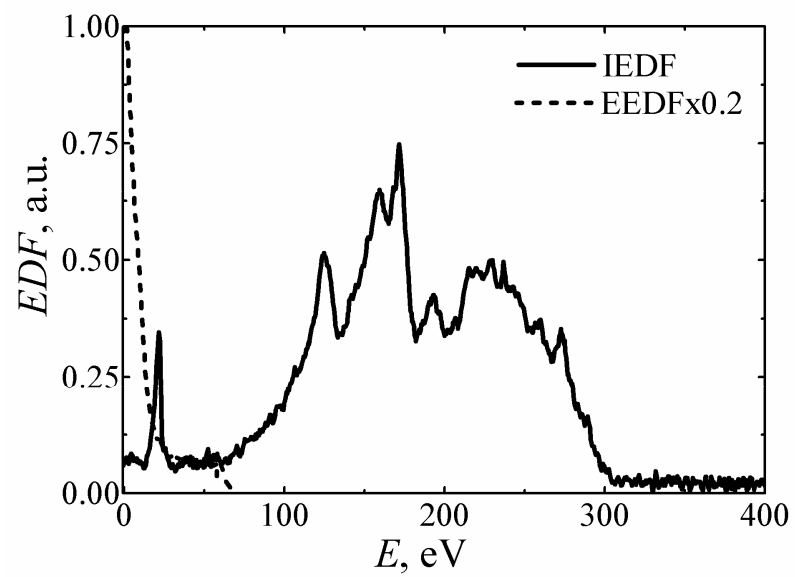

Fig. 12. The IEDF and EEDF of the ion-electron beam generated using nitrogen as a working gas.

The possibility to operate the thruster with working gases other than Ar has also been investigated. The IEDF and EEDF of an ion-electron nitrogen beam at an RF voltage of $150 \mathrm{~V}$ is shown on Fig. 12. The IEDF of the nitrogen beam is narrower than the one measured in $\mathrm{Ar}$ (see fig. 6 above). The beam is also here fully compensated by self-emitted electrons, which was checked by measuring the floating potential in the beam and by comparing fluxes measured by the RFEA. The Neptune thruster acceleration scheme is thus apparently insensitive to the operating gas (propellant). We therefore believe that the Neptune thruster can in principle use propellant from the residual fuel remaining in chemical propellant tanks after spacecraft launches, as well as traditional Xe and other inert gases.

Thought a preliminary comparison between the performance of the Neptune prototype using RF and DC acceleration is present above, further work is required to characterize the thruster efficiency (thrust, power, propellant utilization, etc.) and analyze the various advantages and possible drawbacks of the RF acceleration concept. This should include measurements of the total system efficiency and be compared to standard systems using external neutralizers. However, all of this requires manufacturing of the new Neptune prototype similar to existing ion thruster (by power, thrust and specific impulse values) specially dedicated to such specific measurements. 


\section{Conclusion}

In this paper we experimentally show that a DC self-bias can be formed in a two-gridded system, where only the first biased grid is in direct contact with the plasma and the second grounded grid is in contact with the plasma only via the perforation in the first grid. We propose to use this self-bias formation for ion acceleration accompanied by electron co-extraction. In this case electrons arriving from the plasma to the extraction grids within short time intervals of the RF period (when the RF oscillating sheath potential approaches zero) can be used for beam space charge neutralization.

In this paper we present the first thruster prototype based on this RF acceleration concept. This thruster, called Neptune, is a gridded thruster with strong similarities to space proven thrusters already in operation. The originality lays only in the acceleration technology where the grid system is biased with RF power across a capacitor. This allows a DC self-bias to form charging up the blocking capacitor such that ions are continuously accelerated, while electrons are emitted in a short period in the RF cycle when the plasma-grid sheath collapses.

We have here presented the first proof-of-principles. The first thruster prototype is mounted on a moderate sized vacuum chamber. One single RF generator, at $4 \mathrm{MHz}$, is used for gas ionization, ion acceleration and electron extraction, without the need for any additional DC supplies. A well-collimated ion-electron beam is formed with independent control of the ion flux and ion energy. The ion beam energy can reach up to $400 \mathrm{eV}$ in the current system, and is limited at the moment by the available experimental equipment. The measured beam current, spatial distribution and plume properties are similar to results achieved using a traditional scheme with DC biased grids and a downstream electron neutralizer. Hence, for an optimized system the propellant and power efficiency are expected to be in the same range as for common gridded thrusters. Our measurements show also that the potentials induced on insulated surfaces in the plume are lower in the RF mode, compared to the traditional DC mode under otherwise similar conditions.

Since the main modification to existing gridded ion thrusters, and in particular the ones driven by RF, is the way the grid system is biased (RF instead of DC), the rest of the thruster implementation can benefit from technology heritage. Hence, facilitating a rather rapid increase in the TRL (Technological Readiness Level) of this technology.

\section{Acknowledgements}

This work was supported by a Marie Curie International Incoming Fellowships within the 7th European Community Framework (Neptune PIIF-GA-2012-326054). The authors would like to thank Trevor Lafleur for useful discussions and advices.

\section{References}

[1] Goebel D M and Katz I 2008 Fundamentals of Electric Propulsion (Wiley, Hoboken)

[2] Kuninaka Hitoshi, Molina-Morales Pedro 2004 Acta Astronautica 55 P27-38

[3] Nishiyama K. and Kuninaka H., Transactions Of The Japan Society For Aeronautical And Space Sciences, Aerospace Technology Japan Vol. 10 (2012) No. ists28 (ISTS Special Issue: Selected papers from the 28th International Symposium on Space Technology and Science) p. Tb_1-Tb_8

[4] Dudin S V and Rafalskyi D V 2009 EPL 8855002

[5] Bizioukov A A, Kashaba A Y, Sereda K N, Tseluyko A Ph, and Yunakov N N 1996 Review of Scientific Instruments 674117

[6] Ji Q, Ji L, Chen Y, and Leung K-N 2004 Applied Physics Letters 854618

[7] Pottinger S, Lappas V, Charles C and Boswell R 2011 J. Phys. D: Appl. Phys. 44 235201

[8] Arefiev A V and Breizman B N 2004 Phys. Plasmas 112942 
[9] "Electron-Cyclotron-Resonance (ECR) Plasma Acceleration" J. C. Sercel, paper AIAA-87 -1407 from AIAA 19th Fluid Dynamics, Plasma Dynamics and Lasers Conference. June 8-10, 1987 Honolulu, Hawaii.

[10] Chabert P 2008 Electronegative plasma motor US Patent 2008/0271430 A1

[11] Aanesland A, Meige A, and Chabert P 2009 J. Phys.: Conf. Ser. 162012009

[12] Groh K H and Loebt H W 1991 Journal of Propulsion and Power 7573.

[13] Bugrova A I, Bugrov G E, Davydov V A, Desyatskov A V, Kozintseva M V, Lipatov A S, Safronov A A, Smirnov P G, Kharchevnikov V K, Shaposhnikov M I, Pil'nikov A V 2014 Technical Physics Letters $\mathbf{4 0} 81$

[14] Dudin S V, Rafalskyi D V and Zykov A V 2010 Rev. Sci. Instrum. 81083302

[15] Lieberman M A And Lichtenberg A J 2004 Principles of Plasma Discharges and Materials Processing, 2nd ed. (Wiley, New York)

[16] Dudin S V and Rafalskyi D V 2012 Rev. Sci. Instrum. 83113302

[17] Coburn J. W. and Kay E. 1972 J. Appl. Phys. 434965

[18] Köhler K., Coburn J. W., Horne D. E., Kay E., and Keller J. H. 1985 Journal of Applied Physics 5759

[19] Rafalskyi D, Aanesland A 2014 Dispositif de formation d'un faisceau quasi-neutre de particules de charges opposées French patent application No. 1453469 filed 17 April 2014

[20] Gahan D, Daniels S, Hayden C, O’ Sullivan D and Hopkins M B 2012 Plasma Sources Sci. Technol. 21015002

[21] Rafalskyi D and Aanesland A 2013 EPL 10435004

[22] Rafalskyi D, Popelier L and Aanesland A 2014 J. Appl. Phys. 115053301

[23] Bredin J., Chabert P., and Aanesland A. 2013 Appl. Phys. Lett. 102154107

[24] Kawamura E, Vahedi V, Lieberman M A and Birdsall C K 1999 Palsma Sources Sci. Technol. 8 R45

[25] Wild C and Koidl P 1991 J. Appl. Phys. 692909

[26] Fancey K S and Matthews A 1987 Surf. Coat. Technol. 3317

[27] Hagelaar G J M and Pitchford L C 2005 Plasma Sources Sci. Technol. 14722

[28] Randolph T., Kim V., Kaufman H. R., Kozubsky K., Zhurin V. V. et al., "Facility Effects on Stationary Plasma Thruster Testing," in 23rd International Electric Propulsion Conference, Seattle, WA, Sept. 13-16, 1993. 